\title{
Evaluating Smart PSS Solutions with Context-Awareness in Usage Phase
}

\author{
Zuoxu WANG ${ }^{\mathrm{a}, 1}$, Xinyu LI ${ }^{\mathrm{a}, \mathrm{b}}$, Chun-Hsien $\mathrm{CHEN}^{\mathrm{a}}$ and Li-Pheng $\mathrm{KHOO}^{\mathrm{a}}$ \\ a School of Mechanical and Aerospace Engineering, Nanyang Technological \\ University, Singapore 639798 \\ ${ }^{\mathrm{b}}$ Delta-NTU Corporate Laboratory for Cyber-Physical System, School of Electrical \\ and Electronic Engineering, Nanyang Technological University, Singapore 639798
}

\begin{abstract}
With the mature development of IoT techniques, smart PSS emphasizes the digital transformation of the traditional PSS, which is an ever-evolving and value-co-creation system with the participation of users through the whole product lifecycle, especially during the usage stage. Due to the frequent changes of usage situations, the smart PSS providers are supposed to evolve their product-service bundles according to the trends of usage situations. It is critical to determine what is the current uasge situation, thereafter to evaluate and select proper product-service bundles during the usage phase. In this article, we propose a context-aware approach to evaluate the smart PSS performance during the usage phase.
\end{abstract}

Keywords. Product-service systems, Smart Product-Service System (Smart PSS), solution evaluation, sustainability, context-awareness

\section{Introduction}

In the era of digitalization, the traditional manufacturing companies have been looking for the way to extend their business scope to a integration of both physical products and intangible services, i.e. Product-service systems (PSS) [1]. PSS has the essential features that it is a multi-dimensional system including various actors and diverse product-service bundles [2], making the evaluation of PSS a challenging task. As Mourtzis, et al. [3] stated, PSS evaluation approaches were not be fully explored since lots of emphases were given to the design methodology of PSS. Only about one fifth (18\%) related academic researches have mentioned the importance of PSS evaluation from 1999 to 2016 [3]. Furthermore, only a small portion of companies can get a profit in their business transformation [2]. One of the challenges of the PSS development is to make sufficient evaluation with the consideration of diverse stakeholder's interests before and after launching it to the market.

Furthermore, with the mature development of IoT techniques, a new paradigm of PSS, i.e. smart PSS, has appeared [4]. It emphasizes the digital transformation of the traditional PSS, which is an ever-evolving and value-co-creation system with the participation of users through the whole product lifecycle, especially during the usage stage [5]. However, due to the frequent change of the usage situations, the smart PSS

\footnotetext{
${ }^{1}$ Corresponding Author, Mail: zuoxu001@e.ntu.edu.sg.
} 
providers are supposed to evolve their product-service bundles according to the changes of usage situations $[6,7]$.

Facing to the above problems of complex system evaluation and the change of usage situations, it is critical to determine the current usage situation for a user and thereafter select proper product-service bundles during the usage phase. In this article, we proposed a context-aware approach to evaluate the smart PSS performance during the usage phase. The rest of this paper is organized as follows. Section 1 discussed the existing studies about smart PSS and solution evaluation in the field of PSS. The proposed context-aware smart PSS evaluation method is described in Section 2. Then we give an illustrative example of evaluating the performance of an online 3D printing rent website in Section 3. Finally, the conclusions are summarized in Section 4.

\section{Literature review}

\subsection{Smart PSS and context-awareness in smart PSS}

Smart PSS is a multi-disciplinary and integrated system containing smart products/services, various stakeholders and associated platform, which was firstly proposed by Valencia Cardona, et al. [4]. The term 'smart' generally means the ability of making smart/wise decisions based on the data from networked smart products and services for generating/upgrading the functionalities [8]. Compared with the traditional PSS, it emphasize several features in the literature, such as high degree of autonomy [8] (e.g., real-time reaction [8] and context-awareness [4]), high connectedness [9], degree of personalization [9] and value-co-creation [10].

Particularly, the importance of contexts has been highlighted in providing correct value propositions or functionalities by the participants of a survey [4]. More specically, the end-users are expecting unique rather than generalized functionalties which are dependent of contexts from the smart PSS teams. A context can be defined as any information that can be used to characterize the situation of an entity [11]. An entity is a person, place, or object that is considered relevant to the interaction between a user and an application, including the user and applications themselves . In this study, the contexts refer to the end-users' usage situations instead of the design process situations. Following the idea of context-awareness in smart PSS, the service providers are expected to upgrade their functionalities or give rapid reactions once the contexts of the end-users change.

\subsection{Solution evaluation in PSS}

The assessment of PSS is usually measured by key performance indicators (KPIs) in terms of the diverse evaluation objectives for different stakeholders [2]. According to the indicators used in the evaluation process, PSS can be evaluated from three perspectives, including customer, sustainability, risk and so on [3].

As for the indicators based on customer perceptions, the studies mainly focus on the early design stage. Kimita, et al. [12] have identified PSS features with relation to customer satisfaction and proposed a non-linear satisfaction-attribute (S-A) function to quantify customer satisfaction. Lee, et al. [13] concentrated on assessing the probability of acceptance of new PSS concepts by users. Analytic Network Process (ANP) and Niche Theory were used by them to quantify customer acceptance. Though lots of evaluation tools have been tested and used, the evaluation of PSS is still non-comprehensive by 
considering the process with only one type of stakeholder (i.e. customers) simply. Furthermore, the other phases of PSS, such as manufacturing, logistic and especially usage phase, are omitted.

Another perspective in PSS solution evaluation focuses on sustainability, which is the hotspot in recent studies. It assesses PSS from three dimensions of environmental, social and economic aspects [14], thus making the evaluation more comprehensive. Sun, et al. [15] studied the interrelationships between the PSS's provider and accepter (i.e. customers) and thereafter designed five factors to evaluate the product-service performance, including time, quality, cost, stability and reliability. Similarly, an evaluation scheme for PSS was designed by Kim, et al. [16] considering both providers and the customers as well. The evaluation criteria contain profitability, planet, people, quality and cost.

Other studies also discussed the influence of risk. Abramovici, et al. [17] introduced an indicator framework to monitor the PSS in the usage phase. In their framework, product quality (such as reliability, stability), service quality (such as service reliability, service assurance, responsiveness) and cooperation quality have been considered.

Based on the reviewed literature, the performance or quality evaluation of PSS mainly concentrates on the indicators of the customer perceptions in the early design phase and manufacturing process before launching PSS provision to the market. However, smart PSS intends to achieve smart decisions and automatic actions based on various scenarios, a context-aware solution evaluation approach in usage phase is still lacking. The research questions that how to justify the usage scenarios in smart PSS and how to evaluate the possible solutions based on the context information still remains to be explored.

\section{Methodology}

This section describes the overall context-aware smart PSS evaluation approach with details, including the context modeling in PSS and the solution evaluation framework. In the first half, the context features will be predefined by experts/engineers, their patterns will be extracted and categorized as diverse scenarios. Afterward, compared with the conventional PSS evaluation approach conducted in the early design stage, a context-aware solution evaluation module is added and studied.

\subsection{Context modeling and scenario identification}

The basis for context-aware applications is a well-defined context model [18]. Based on the widely accepted context model defined in [19], three core steps are designed to adapt context-awareness into smart PSS, namely (1) predefine the context features of interest; (2) identify the scenarios of interest and (3) detect scenarios of interest. In this research, we follow the key steps of building up a context-aware system, as shown in Figure 1. 


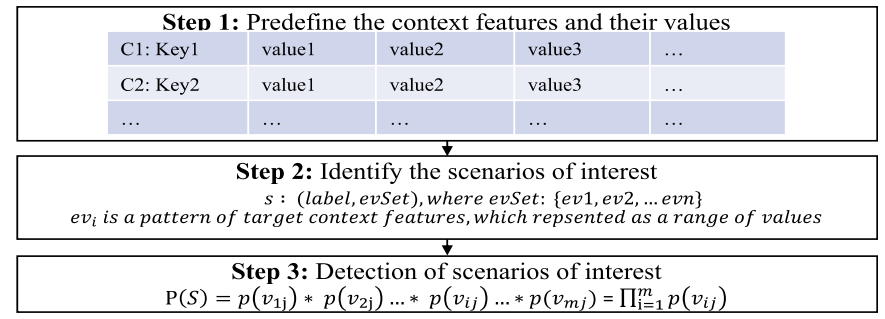

Figure 1. An overview of the context modeling and scenario identification.

\subsubsection{Predefine the context features of interest}

To achieve context-aware applications, it is necessary to define the involved entities with the variables and relationships in the environment [20]. Key-value modeling [21] is applied to represent each context features. A context feature can be defined as a set which has a key and the set of possible values, denoted as

$$
C_{i}=\left(k e y,\left\{v_{1}, v_{2}, \ldots\right\}\right) .
$$

The aggregation of context features can be expressed as a set of context features from various context categories, which is denoted as $C=\left\{C_{1}, C_{2}, \ldots, C_{m}\right\}$ where $m$ means the number of context features. The concept of semantic relations $\mathrm{R}$, as defined in [19], is used to identify the relationship/behaviors among the context features. For example, some semantic relations can be defined as

$$
\begin{gathered}
<3 \text { DPrinter, hasModel, productModel }> \\
<3 \text { PPrinter, hasSensor, nozzleTemperature }>\text {, } \\
<3 \text { DPrinter, is locatedAt, location }> \\
<\text { User, isLocatedAt, location }> \\
<\text { UserA, hasPreference, productModel }>
\end{gathered}
$$

\subsubsection{Identify the scenarios of interest}

After defining the context models and their relationship, it is possible to identify scenarios of interest in smart PSS. A scenario refers to the current situation of the product-service bundles and its involved environment, which can be represented as a tuple with its label and a set of events [20]. A mathematical expression of the scenario is

$$
s:(\text { label, evSet) }
$$

, where evSet refers to the set of one or more predefined contexts with values, that

$$
\text { evSet: }\left(C_{1}: v_{1}, C_{2}: v_{2}, \ldots, C_{n}: v_{n}\right) \cap \text { Relation }\left\{C_{i}\right\} \text {. }
$$

\subsubsection{Detect scenarios of interest}

The scenarios are classified as default scenarios and deflected scenarios in this approach. The default scenarios are preset in the smart PSS that the designers/engineers assume them will happen with a certain pattern while user using the product-service bundles, while the deflected scenarios are the ones whose event sets have been changed from the default scenarios to other patterns. 
In this step, we intend to judge whether an unlabeled scenario is deflected or not. Given a new unlabeled scenario, we would like to identify either it belongs to a predefined scenario or not based on its context features' values. Here we set the probability of context feature $C_{i}$ 's value appearing in positive scenarios as $p\left(v_{i j}\right)$. Each involved context features may have their own probability distribution, such as Bernouli distribution, binomial distribution, Poisson distribution, normal distribution and so on With the assumption that context feature is independent with each other, the probability of a scenario is a positive one can be computed by using the following equation:

$$
\mathrm{P}(S)=p\left(v_{1 \mathrm{j}}\right) * p\left(v_{2 \mathrm{j}}\right) \ldots * p\left(v_{i j}\right) \ldots * p\left(v_{m j}\right)=\prod_{\mathrm{i}=1}^{m} p\left(v_{i j}\right)
$$

, where $S i$ is the unlabelled scenario, $v_{i j}$ is the $j$-th value of context feature $C_{i}$ appearing in a positive scenario. If $\mathrm{P}(S)$ is larger than the threshold $\varepsilon$, then it is a default scenario that is initially desired by the users. Otherwise, it belongs to a deflected scenario.

\subsection{Solution evaluation framework}

If a deflected scenario is detected, solutions should be evalauted based on the interests of both the users and service providers/manufacturers, then the proper solution will be selected to response to the scenario change. The evaluation objective and the evaluation indicators need to be identified.

Inspired by the concept of customer value $(\mathrm{CV})$ which is formulated by Reidenbach [22], denoted as $C V=\frac{\text { Utility }}{\text { cost }}$, a novel evaluation objective, i.e. stakeholder value (SV) which expands the evaluation scope to measure the total utility of smart PSS with the consideration of interests of both customers and manufacturers, was proposed. It is the objective function for the aim of maximizing the value/interest of both customers and manufacturer/service providers. To achieve it, five relative evaluation criterion are defined as follows.

1. maximize the smart PSS quality $(\boldsymbol{Q})$;

2. minimize the cost $(\boldsymbol{C})$;

3. maximizing the customer satisfaction $(\boldsymbol{C S})$;

4. maximize (at least remain) the sustainability $(\boldsymbol{S})$ of smart PSS platform; and

5. maximize the value-co-creation $(\boldsymbol{V C C})$ capability.

Hence the abovementioned objective function $\boldsymbol{S} \boldsymbol{V}$ can be formulated as

$$
S V=\frac{w_{1} * Q+w_{2} * C S+w_{3} * S+w_{4} * V C C}{C}
$$

Smart PSS quality $(Q)$. Initially, the quality characteristics which affect the functions of smart PSS should be identified. Those characteristics can be selected among the context features from the preliminary work. For example, the nozzle flow rate of the $3 \mathrm{D}$ printers is one of the context features and simultaneously a quality characteristic affecting its functional performance as well. The selection of appropriate quality characteristics requires solid engineering knowledge about the products and/or services under investigation. After identifying the quality characteristics, Taguchi's quality loss function [23] is applied to calculate the functional performance for each quality factor. Based on different types of expected quality factors' value, the functional performance can be evaluated as follows. 


$$
L(x)=\left\{\begin{array}{c}
k x^{2}, x \text { is the smaller the better } \\
k(x-m)^{2}, x \text { is nominal }- \text { the best } \\
k \frac{1}{x^{2}}, x \text { is the larger the better }
\end{array}\right.
$$

, where $L(x)$ is the loss function, $K$ means the quality loss coefficient, $x$ is the quality characteristic and $\mathrm{m}$ refers to the expected value when the quality loss is nominal the best. The total quality loss can be calculated as the accumulation of all the quality loss values, i.e. $L_{t}(X)=\sum L(\mathrm{x})$. The total functional performance of a product-service bundle can be determined by

$$
Q=1-\alpha L_{t}(X)=1-\alpha \sum L(x)
$$

, where $\alpha$ is the regularisation coefficient to standardise the value of $Q$ within the range of $0-1$.

Customer satisfaction (CS). A five-point scale ranging from 'dissatisfied' to 'satisfied' is applied to derive customer satisfaction. Here, we focus on the increment of the customer satisfaction value after a solution is provided to the user. To standardise the $\mathrm{CS}$ indicator, the CS is defined as $\mathrm{CS}=\frac{\Delta C S}{C S_{0}}=\frac{C S_{1}-C S_{0}}{C S_{0}}$, where $C S_{0}$ is the original customer satisfaction level and $C S_{1}$ is the customer satisfaction level after receiving a certain solution. The customer satisfaction data can be collected through historical questionnaires on similar product-service bundles.

Sustainability $(\boldsymbol{S})$ of product-service bundles. This study concentrates on the environmental effect together with the economic effect, especially the extended lifespan of product-service bundles and the cost of part reusability in the closed-loop of smart PSS. The sustainability of a product-service bundle in a smart PSS is expressed as

$$
\text { sus }=\frac{\sum \Delta \text { lifespan }_{i}}{\sum \text { lifespan }_{i}}
$$

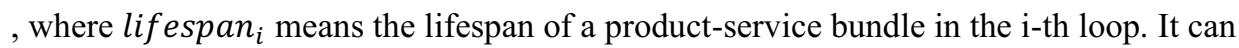
be obtained from the manufacturer/factory of each product modules, which are tested before the product modules are moved to the next factory. The numerical values for $\Delta$ lifespan $_{i}$ can be obtained from the service provider during the operation of the product-service platform. The variable sus indicates the sustainability capacity of a product-service bundle in several update loops, from the perspective of value-in-use retention capacity and extended lifespan.

Value co-creation (VCC) measurement through interaction and personalization. The foundational premises addressed in the S-D logic literature [24] have addressed that value is co-created by multiple actors, including the beneficiary, moreover, value is always uniquely and phenomenologically determined by the beneficiary. Hence, the measurement of value co-creation is related to the interaction between actors (e.g. users and service providers) and the personalization. In this paper, since we focus more on the generated values with the participation of users, only two attributes are adopted to measure the value co-creation capability of smart PSS, i.e., the interaction between users and stakeholders, and personalization. We describe it with the following function:

$$
\begin{aligned}
& v c c=\text { interaction }+ \text { personalization } \\
& \text { interaction }=\frac{\text { no.of interactions }}{\text { no.of interaction channels }}
\end{aligned}
$$




$$
\text { personalization }=\frac{\text { no.of personalized } \text { parts } / \text { modules }}{\text { no.of total } \text { parts } / \text { modules }}
$$

Besides the evaluation indicators, the weights of the evaluation indicators are caseby-case in different PSS application domains. For example, the weights in the aeroplane services are different with the ones in vehicle rent. Usually, the weights in a certain PSS application domain will not change frequently, thus they can be predefined by the experts or obtained through survey from a group of users before the smart PSS solution evaluation. Once triggered by the deflected scenarios, the smart PSS solution can be evaluated according to the described equations considering the needs of multiple stakeholders. The larger the $S V$ is, the better the performance of the solution will be.

\section{An illustrative example of remote $3 D$ printing system}

This section utilizes a remote $3 \mathrm{D}$ printing system which mainly offers the online 3D printing services and associate services (e.g., 3D printer phase recommendation and discussion forum) as an illustrative example to validate the feasibility of the proposed approach. The 'smartness' of this example is reflected in the automatic scenario identification and the solution process once the scenario changes. In this example, an individual user whose usual behavior is ordering the 3D printing services online regularly and intermittently (default scenario) is set as the given user.

\subsection{Context modeling and scenario identification of the remote $3 D$ printing system}

For the described scenarios, several context features are collected and summarized in Table 1 . The value boundaries of each context features are decided based on the domain knowledge which collected from a 3D printing website: 3 dhubs.com.

Table 1. Context features and their values of the remote $3 \mathrm{D}$ printing system.

\begin{tabular}{ccc}
\hline $\begin{array}{c}\text { Context } \\
\text { feature } \\
\text { No. }\end{array}$ & $\begin{array}{c}\text { Context feature name } \\
\text { (key) }\end{array}$ & Values of context features \\
\hline $\mathrm{C} 1$ & printFrequencyMonthly & $\{1: 0-5 ; 2: 10-25 ; 3:>=25\}$ \\
\hline $\mathrm{C} 2$ & printDurationOneTime & $\{(0,1):<5 \mathrm{~h} ;(1,0):>=5 \mathrm{~h}\}$ \\
\hline $\mathrm{C} 3$ & modelSize & $\{(0,1):<=400 * 400 * 400 \mathrm{~mm} ;(1,0):>400 * 400 * 400 \mathrm{~mm}\}$ \\
\hline $\mathrm{C} 4$ & user & $\{(0,1):$ new user $($ time after registration is less than 3 months $) ;$ \\
& & $(1,0):$ regular user $($ else $)\}$ \\
\hline
\end{tabular}

At the same time, the semantic relations between the defined context features include but not limited to:

$$
\begin{aligned}
& <\text { user, hasPreference, printFrequencyMonthly }> \\
& <\text { modelSize, identify, printDurationOneTime }>
\end{aligned}
$$

The value of modelSize, printFrequencyMonthly are decided by users. The value of printDurationOneTime is represented by the modelSize, while the model is still offered by the users themselves. Based on the context features and their relations, one default scenario and two deflected scenarios of interest are represented in Table 2, including 'Regular printing intermittently', 'Frequent printing' and 'Long continuous printing'. 
Furthermore, the scenarios depend on three context features, i.e. user, printFrequencyMonthly and printDurationOneTime. Their value ranges and decision boundaries are predefined as Table 3 shown. The probability distribution functions of them are defined as follows. Context feature user follows a sigmoid function, printFrequencyMonthly and printDurationOneTime follow the Poisson distribution.

$$
\begin{gathered}
\mathrm{P} 1=\mathrm{P}\{\mathrm{C} 4=(0,1)\}=\frac{1}{1+e^{-(-t+3)}} \\
\mathrm{P} 2=\mathrm{P}\{\mathrm{C} 1=\mathrm{k}\}=\frac{17^{k} e^{-17}}{k !} \\
\mathrm{P} 3=\mathrm{P}\{\mathrm{C} 2=\mathrm{k}\}=\frac{2.5^{k} e^{-2.5}}{k !}
\end{gathered}
$$

In this way, the probability of a scenario is a default scenario can be calculated as

$$
\mathrm{P}(\text { default scenario })=P 1 * P 2 * P 3
$$

\begin{tabular}{|c|c|c|c|c|}
\hline $\begin{array}{c}\text { Scenario } \\
\text { No. }\end{array}$ & Scenario name & evSet & Pattern & Scenario type \\
\hline S1 & $\begin{array}{l}\text { Regular printing } \\
\text { intermittently }\end{array}$ & $\{\mathrm{ev} 1\}$ & $\begin{array}{c}\text { \{user: }(1,0) \cap \\
\text { printFrequencyMonthly: } 2 \cap \\
\text { printDurationOneTime: }(0,1)\}\end{array}$ & $\begin{array}{l}\text { Default usage } \\
\text { scenarios }\end{array}$ \\
\hline $\mathrm{S} 2$ & Frequent printing & $\{\mathrm{ev} 2\}$ & $\begin{array}{l}\text { \{user: }(0,1) \\
\cap \text { (printFrequencyMonthly: } 1 \\
\cup \text { printFrequencyMonthly: } 3) \\
\cap \text { printDurationOneTime: }(0,1)\}\end{array}$ & $\begin{array}{c}\text { Deflected } \\
\text { usage scenario }\end{array}$ \\
\hline S3 & $\begin{array}{l}\text { Long continuous } \\
\text { printing }\end{array}$ & $\{\mathrm{ev} 3\}$ & $\begin{array}{l}\text { \{user: }(0,1) \\
\cap \text { (printFrequencyMonthly: } 1 \\
\cup \text { printFrequencyMonthly: } 3) \\
\cap \text { printDurationOneTime: }(0,1)\}\end{array}$ & $\begin{array}{c}\text { Deflected } \\
\text { usage scenario }\end{array}$ \\
\hline
\end{tabular}

The threshold was set as 0.05 since the involved scenarios will not significantly cause a breakdown or other security accidents that need a lower threshold.

Table 2. The scenarios of interest.

Table 3. Default values or intervals and optimal values for the involved context features.

\begin{tabular}{cccc}
\hline Context features & Default values & Default intervals & Optimal values \\
\hline $\begin{array}{c}\text { user (time length after registration, } \\
\text { month) }(\mathrm{C} 4)\end{array}$ & New user:(0,1) & $0-3$ & $/$ \\
$\begin{array}{c}\text { printFrequencyMonthly (times) }(\mathrm{C} 1) \\
\text { printDurationOneTime }(\mathrm{h})(\mathrm{C} 2)\end{array}$ & Regularly:2 & $10-25$ & 17 \\
\hline
\end{tabular}

\subsection{Solution evaluation of the remote $3 D$ printing system}

During the usage phase of the remote $3 \mathrm{D}$ printing system, if the usage scenarios are detected deflected from the default scenario (i.e. S1 : Regular printing intermittently), some certain solutions should be conducted. Assume that two solutions are the alternative solution, namely Recommend user to buy a printer and Recommend user to change parameters.

According to the equation (5), we can evaluate the two solutions, as shown in Table 4. Each evaluation indicators are evaluated and normalized into a range of $[0,1]$. Though 
a survey of the $3 \mathrm{D}$ printing experts and end-users, the weights of the evaluation indicators were derived as $[0.29,0.37,0.21,0.13]$. Table 4 shows the values of each evaluation indicators for each solution. The results indicate that given scenario 2 (S2), Solution 2 (Sol1) has a higher SV value, meanwhile give scenario 3 (S3), Solution 3 (Sol3) has the higher SV value.

Table 4. Evaluation results of short-term solutions.

\begin{tabular}{ccc}
\hline & Sol1: Recommend user to buy a printer & Sol2: Recommend user to change parameters \\
\hline $\mathrm{S} 2$ & $\mathrm{Q}=0.508, \mathrm{CS}=0.893, \mathrm{~S}=0.581$ & $\mathrm{Q}=0.239, \mathrm{CS}=0.117, \mathrm{~S}=0.320$, \\
& $\mathrm{VCC}=0.526, \mathrm{C}=0.881$ & $\mathrm{VCC}=0.181, \mathrm{C}=0.283$ \\
$\mathrm{SV}=0.762$ & $\mathrm{SV}=0.711$ \\
\hline $\mathrm{S} 3$ & $\mathrm{Q}=0.087, \mathrm{CS}=0.346, \mathrm{~S}=0.559$, & $\mathrm{Q}=0.887, \mathrm{CS}=0.510, \mathrm{~S}=0.903$, \\
& $\mathrm{VCC}=0.081, \mathrm{C}=0.881$ & $\mathrm{VCC}=0.645, \mathrm{C}=0.283$ \\
$\mathrm{SV}=0.317$ & $\mathrm{SV}=2.528$ \\
\hline
\end{tabular}

\section{Conclusion}

Though lots of studies have discussed the evaluation of PSS, context-awareness as one of the unique features of smart PSS has not been deeply discussed. The contributions of this work are summarized as follow. First, this approach defined the manner of both context features and scenarios in smart PSS, meanwhile offered an operable approach to justify whether a scenario has changed or not. The approach of context modeling and scenario identification serves as a foundation in smart PSS to assist the decision-makings in further design activities, making the solution evaluation more autonomous. Furthermore, a novel evaluation objective SV was proposed. It expand the evaluation scope from just customer value to the value of both customers and stakeholders, making the smart PSS be more sustainable and value-co-created.

Except for the contributions, the limitations of this research appeared as well. The decision boundaries of context features now depend on the domain knowledge, which need to be set by experts manually. A decision boundary learning approach remains further study.

\section{Acknowledgement}

This work was conducted within the Delta-NTU Corporate Lab for Cyber-Physical Systems with funding support from Delta Electronics Inc. and the National Research Foundation (NRF) Singapore under the Corp Lab@University Scheme. And it is supported by Fraunhofer Singapre.

\section{References}

[1] M. J. Goedkoop, C. J. G. Van Halen, H. R. M. Te Riele, and P. J. M. Rommens, "Product service systems, ecological and economic basics," Report for Dutch Ministries of environment (VROM) and economic affairs (EZ), 1999, vol. 36, no. 1, pp. 1-122.

[2] D. Chen, X. Chu, X. Yang, X. Sun, Y. Li, and Y. Su, PSS solution evaluation considering sustainability under hybrid uncertain environments, Expert Systems with Applications, vol. 42, no. 14, pp. 5822-5838, 20152015.

[3] D. Mourtzis, M. Doukas, and S. Fotia, Classification and mapping of PSS evaluation approaches, IFAC-PapersOnLine, vol. 49, no. 12, pp. 1555-1560, 20162016. 
[4] A. M. Valencia Cardona, R. Mugge, J. P. L. Schoormans, and H. N. J. Schifferstein, Challenges in the design of smart product-service systems (PSSs): Experiences from practitioners, in Proceedings of the 19th DMI: Academic Design Management Conference. Design Management in an Era of Disruption, London, UK, September 2-4, 2014, 2014 2014, London, UK: Design Management Institute.

[5] P. Zheng, Z. Wang, C.-H. Chen, and L. P. Khoo, A survey of smart product-service systems: Key aspects, challenges and future perspectives, Advanced engineering informatics, vol. 42, p. 100973, 2019.

[6] Z. Wang, C.-H. Chen, P. Zheng, X. Li, and L. P. Khoo, A novel data-driven graph-based requirement elicitation framework in the smart product-service system context, Advanced engineering informatics, vol. 42, p. 100983, 2019, doi: https://doi.org/10.1016/j.aei.2019.100983.

[7] C. K. Chang, H. Jiang, H. Ming, and K. Oyama, Situ: A Situation-Theoretic Approach to ContextAware Service Evolution, IEEE Transactions on Services Computing, vol. 2, no. 3, pp. 261-275, 2009, doi: 10.1109/TSC.2009.21.

[8] B. Kuhlenkötter et al., New Perspectives for Generating Smart PSS Solutions - Life Cycle, Methodologies and Transformation, (in en), Procedia CIRP, vol. 64, pp. 217-222, 2017, doi: 10.1016/j.procir.2017.03.036.

[9] P. Zheng, X. Xu, and C.-H. Chen, A data-driven cyber-physical approach for personalised smart, connected product co-development in a cloud-based environment, Journal of Intelligent Manufacturing, pp. 1-16, 2018.

[10] Z. Liu, X. Ming, W. Song, S. Qiu, and Y. Qu, A perspective on value co-creation-oriented framework for smart product-service system, (in en), Procedia CIRP, vol. 73, pp. 155-160, 2018, doi: 10.1016/j.procir.2018.04.021.

[11] G. D. Abowd, A. K. Dey, P. J. Brown, N. Davies, M. Smith, and P. Steggles, Towards a Better Understanding of Context and Context-Awareness, in Handheld and Ubiquitous Computing, Berlin, Heidelberg, H.-W. Gellersen, Ed., 1999: Springer Berlin Heidelberg, pp. 304-307.

[12] K. Kimita, Y. Shimomura, and T. Arai, Evaluation of customer satisfaction for PSS design, Journal of Manufacturing Technology Management, vol. 20, no. 5, pp. 654-673, 20092009.

[13] S. Lee, Y. Geum, S. Lee, and Y. Park, Evaluating new concepts of PSS based on the customer value: Application of ANP and niche theory, Expert Systems with Applications, vol. 42, no. 9, pp. 4556-4566, 2015.

[14] D. Mourtzis, S. Fotia, E. Vlachou, and A. Koutoupes, A Lean PSS design and evaluation framework supported by KPI monitoring and context sensitivity tools, The International Journal of Advanced Manufacturing Technology, vol. 94, no. 5-8, pp. 1623-1637, 2018.

[15] H. Sun, Z. Wang, Y. Zhang, Z. Chang, R. Mo, and Y. Liu, Evaluation method of product-service performance, International Journal of Computer Integrated Manufacturing, vol. 25, no. 2, pp. 150$157,20122012$.

[16] K.-J. Kim, C.-H. Lim, J.-Y. Heo, D.-H. Lee, Y.-S. Hong, and K. Park, An evaluation scheme for product-service system models with a lifecycle consideration from customer's perspective, in Reengineering Manufacturing for Sustainability: Springer, 2013, pp. 69-74.

[17] M. Abramovici, F. Jin, and H. B. Dang, An indicator framework for monitoring IPS ${ }^{2}$ in the use phase, in Product-service integration for sustainable solutions: Springer, 2013, pp. 311-322.

[18] D. Stokic and A. T. Correia, Context sensitive Web service engineering environment for product extensions in manufacturing industry, Service Computation 2015, pp. 9-13, 20152015.

[19] A. Machado, V. Maran, I. Augustin, L. K. Wives, and J. P. M. de Oliveira, Reactive, proactive, and extensible situation-awareness in ambient assisted living, Expert Systems with Applications, vol. 76, pp. 21-35, 2017.

[20] M. S. Teixeira, V. Maran, J. P. M. de Oliveira, M. Winter, and A. Machado, Situation-aware model for multi-objective decision making in ambient intelligence, Applied Soft Computing, p. 105532, 20192019.

[21] M. Samulowitz, F. Michahelles, and C. Linnhoff-Popien, Capeus: An architecture for contextaware selection and execution of services, in IFIP International Conference on Distributed Applications and Interoperable Systems, 2001 2001: Springer, pp. 23-39.

[22] R. E. Reidenbach, Dominating Markets with Value: Advances in Customer Value Management. Rhumb Line, 2002.

[23] G. Taguchi. (1993, 1993/03//) Robust technology development. Mechanical Engineering-CIME [Article]. 60+.

[24] R. F. Lusch and S. L. Vargo, "Service-dominant logic: reactions, reflections and refinements," Marketing theory, vol. 6, no. 3, pp. 281-288, 2006. 